\title{
$\angle S$ Research Square \\ Effect of RPR titer of pregnant syphilis on adverse outcome and treatment in 2013-2018, Nanjing, China: a retrospective study
}

Genju Wang ( $\square$ wanggenju@sina.com )

The second Affiliated hospital of the Southeast University https://orcid.org/0000-0003-2310-4729 Ying Ji

The second affiliated hospital of the southeast university

\section{Guorong Han}

The second affiliated hospital of the southeast university https://orcid.org/0000-0002-1397-8185

\section{Hongxiu Jiang}

The second affiliated hospital of the southeast university https://orcid.org/0000-0001-7914-5875

\section{Juan Tang}

The second affiliated hospital of the southeast university

\section{Yi Ding}

The second affiliated hospital of southeast university

\section{Research article}

Keywords: Syphilis infected pregnancy; Adverse outcomes; Congenital syphilis; Benzathine penicillin G; RPR titer

Posted Date: March 9th, 2020

DOl: https://doi.org/10.21203/rs.3.rs-16308/v1

License: (9) This work is licensed under a Creative Commons Attribution 4.0 International License. Read Full License 


\section{Abstract}

Background: We aimed to find the relationship among syphilis Rapid Plasma Reagin (RPR) titer of syphilis infected pregnant women, RPR positive rate of the newborn, the adverse pregnancy outcomes and therapies.

Method: A total of 600 syphilis infected pregnant women and their infants were participated in our study. Syphilis RPR Test and Treponema pallidum particle agglutination (TPPA) test screening were performed on mothers and these infants. Mothers were divided into six groups depending on different treatments.

Results: The higher the RPR titer of the pregnant mother was, the higher the RPR titer of their newborns after birth. The number of newborns in double negatives (RPR negative and TPPA negative), TPPA positive (RPR negative), RPR $<8$ and RPR $\geq 8$ groups $(P<0.01)$ has significantly difference. The infants with TPPA positive and RPR $<1: 8$ have more possibility to turn double negative than those RPR $\geq 8$ infants after 12 months. Among all the adverse events, the number of premature births was significantly higher than others $(P<0.01)$. The proportion of adverse events achieved highest in RPR $\geq 1: 8$ group (11.24\%), compared with the TPPA positive group (8.41\%) and RPR $<1: 8$ group (2.56\%). Therapy efficacy of mothers was better in RPR $\geq 1: 8$ group than RPR $<8$ and TPPA positive groups $(P<0.01)$.

Conclusions: RPR titers of 1:8 could be used as a point of therapeutic induction, which need to be emphasized.

\section{Background}

Syphilis, which is transmitted sexually or from mother to child in utero, is caused by infection with the spirochete Treponema pallidum [1]. In recent years, the incidence of syphilis had gradually increased with the widespread of sexually transmitted diseases, which leading to an increasing number of pregnancy combined with syphilis [2-5]. Treponema pallidum particle agglutination (TPPA) test and serum Rapid Plasma Reagin (RPR) test have been used as normal methods to detect syphilis in many countries [6-9].

Untreated or inadequately treated syphilis in pregnancy could result in some adverse pregnancy outcome, including stillbirth, premature birth, early fetal loss, neonatal death, low birth weight and congenital syphilis [10]. Not only that, there were many other studies had put focus on the pregnancy outcomes [11, 12]. In a former study of Deborah et al [13] in Tanzania, they revealed that pregnancy women with RPR $\geq$ 1: 8 had a high risk of stillbirth, low-birth-weight and preterm live birth than uninfected women. Zhang et al [14] revealed that the adverse pregnancy outcomes, including congenital syphilis, neonatal deaths, and neonatal asphyxia decreased from 2013 to 2015 in Beijing, China. Some studies have revealed the importance of routine screening for the discovery and treatment of syphilis infected pregnancy women [15]. However, there were still not enough researches had put focus on the relationship among RPR titer of syphilis infected pregnant women with RPR positive rate of the newborn and the adverse pregnancy outcomes. 
Benzathine penicillin $\mathrm{G}$ has been recommended as the main treatment for syphilis recent years [16], with zero penicillin-resistance case had been reported in maternal syphilis until now [17-19]. In China, the usual effective recommendation is two courses of penicillin treatment [1], but one course of treatment before gestational week of 28 [20]. However, the penicillin could not been used in all individuals at the conditions of allergic reaction, HIV co-infection and neurosyphilis [21]. Ceftriaxone is recommended as a promising alternative treatment of syphilis for its well tolerance, good central nervous system penetration and effectiveness for neurosyphilis [22].

Nanjing Second Hospital is a Grade III Class A Infectious Disease Specialist Hospital attracting most of the syphilis patients in Nanjing, Jiangsu, China. Based on the detected data $(n=600,2013-2018)$ from the patients, we conducted a retrospective study with the following objectives: (1) to find the RPR titer changes between mothers and their children; (2) to analysis the relationship between RPR titer of mothers and adverse outcome events; (3) to assess the relationship between RPR titer of mothers and the treatment efficacy; and finally (4) we want to provide an important suggestion on RPR titer threshold for treatment and birth.

\section{Methods}

\section{Recruitment of study subjects}

A total of 600 pregnant women (Table 1) with syphilis infection who were treated in Nanjing Second Hospital (infection disease specialist hospital) were detected in January 2013 to December 2018. The age of them mainly distributed between 26 and 35, with an average age of 31.28 (30.79 31.76) years old. A total of 608 newborns (including adverse outcome and 8 twins) were analyzed, with some newborns were followed up for 12 months.

The diagnostic criteria for the syphilis infected pregnant women in this study were: (1) pregnant women or spouses have a history of syphilis infection; (2) the TPPA test and serum RPR test were both positive; (3) have clinical signs and symptoms of the syphilis. The diagnostic criteria for neonatal congenital syphilis are as follows: (1) maternal and neonatal syphilis serological tests are positive, syphilis spirochete continues to rise; or titer is four times higher than its mother; (2) neonatal blood dark field also detects syphilis; (3) newborns have the following two or more clinical features and manifestations: extremity peeling, mucosal damage, macula, hepatosplenomegaly, low body weight, pathological jaundice, dyspnea, edema, ascites, syphilis pseudoparalysis, platelets reduce and anemia. Infants were defined as the delivery of a dead fetus of $>22$ weeks gestation, intrauterine fetal death was defined as a fetal death at $\leq 22$ weeks gestation, and the low baby weight was defined as $<2500 \mathrm{~g}$. Preterm birth was defined as a delivery at $<37$ weeks gestation, and intrauterine growth retardation was defined as a birth weight $<2500 \mathrm{~g}$ with a gestational age of $\geq 37$ weeks.

\section{Syphilis serologic testing}


Serum of pregnant women and the newborns were confirmed including a titrated RPR test and a TPPA test. When both tests were found negative, the samples were judged negative and no further tests were performed as they had no serological sign of a past or an acute syphilis.

\section{Intention-to-treat analysis}

Patients were divided into six groups according to the treatments, they were: (1) 101 syphilis infected pregnant women were treated with benzathine penicillin $\mathrm{G}$ for one course; (2) 18 syphilis infected pregnant women treated with benzathine penicillin $\mathrm{G}$ for one course and cephalosporin for one course; (3) 210 syphilis infected pregnant women were treated with benzathine penicillin G for two courses; (4) 26 syphilis infected pregnant women were treated with benzathine penicillin $\mathrm{G}$ for two course and cephalosporin for two course; (5) 17 syphilis infected pregnant women treated with benzathine penicillin $\mathrm{G}$ for three courses; and (6) there were 53 syphilis infected pregnant women accepted no treatment. Otherwise, there were also some other therapies that were not statistical analyzed in this study. Each course of benzathine penicillin $\mathrm{G}$ was lasted for three weeks, with 2.4 million units in each week with intramuscular injection once diagnosed. Each course of cephalosporin was lasted for ten days with $2.0 \mathrm{~g}$ every day with intravenous drip.

\section{Statistical analysis}

Statistical analyses were performed using IBM SPSS version 22.0 (IBM Corp., Armonk, NY, USA) statistical software or GraphPad Prism v6.04 (GraphPad Software, San Diego, CA, USA). Qualitative data were described by frequency and rate, and statistical data were used for $\chi 2$, Student $t$ tests, as appropriate. $P<0.05$ was defined as significant difference. Rates and $95 \%$ confidence intervals (Cls) were calculated for each measure. Odds rates (ORs) and their $95 \% \mathrm{Cl}$ were used to demonstrate the level of association. Factors including age, newborn weight which associated with APOs at the a level of $5 \%$ in the univariate analysis were included in the multivariable logistic regression.

\section{Results}

A total of 600 syphilis infected pregnant women accepted RPR titers test and TPPA test during the 6-year period in this study. The age of syphilis infected pregnant women was mainly distributed at $26 \sim 30$ (36.83\%), $31 \sim 35(27.17 \%)$, and $>35$ (23.00\%), occupied $87 \%$ out of the 600 patients. The RPR titers of syphilis infected pregnant women were mainly distributed at 1:1 (25.00\%), 1:2 (25.33\%), 1:4 (13.00\%) and TPPA positive (17.17\%). The proportion of RPR $<8$ occupied $63.33 \%$, whereas the RPR $\geq 8$ occupied $19.5 \%$. The RPR titer distributed significantly different among different age groups $(P<0.01)$. The relationship between age distribution and RPR titers of the syphilis infected pregnant women is shown in Table 1.

A total of 514 infants accepted RPR titers and TPPA tests among all the newborns. There were two newborns were tested to be double negative concerns both RPR and TPPA tests. The comparison analysis among mothers revealed that TPPA positive (RPR negative) percentage increased from $17.17 \%$ 
to $41.44 \%$, RPR $<8$ decreased from $63.33 \%$ to $53.11 \%$, and the RPR $\geq 8$ decreased from $19.5 \%$ to $5.06 \%$ (Figure 1a). There were 119 newborns were turned double negative when they were 12 months old, which comprised with 67 lower RPR titers and 52 TPPA positive newborns. There were no newborns with RPR $\geq$ 8 could turn to be double negative. The number of newborns with double negatives, RPR $\geq 8, R P R<8$ and TPPA positive were significantly different in different age groups of their mothers $(P<0.01$, Table 1$)$.

As shown in Table 2, the higher the RPR titer of the pregnant mother was, the higher the RPR titer of the newborn after birth. The RPR titer of the newborns were less than or equal to the titer of their mothers as usual. One of the pregnant mothers in the 1:4 group had a baby with RPR titer of 1:32 after birth, which was eight times than that of its mother, and the RPR did not turn negative after six months, moreover, the RPR of this baby was still 1:8 when it was 12 months years old, which was diagnosed as congenital syphilis. The newborns with TPPA positive or RPR $<1: 8$ have more possibility to turn double negative than those RPR $\geq 8$ ones. The relationship between RPR titer of syphilis before delivery, RPR seropositive rate of the newborns and negative conversion rate after 12 months of born is shown in Table 2.

We have further analyzed the age of infected pregnant women, newborns weight and adverse outcomes events at different RPR titer levels. The result shown that women with higher RPR titer (RPR $\geq 1: 8)$ have a slighter younger age. The newborn weight in RPR $<1: 8$ group was bigger than that in RPR $\geq 1: 8$ group and TPPA positive group (Table 3). One newborn could have one or more outcomes. In the RPR $<1: 8$ group, there were 382 normal newborns and 10 adverse events (nine premature birth and two congenital), among which there was one newborn was both premature birth and congenital. In the RPR $\geq 1: 8$ group, there were 79 normal newborns and 10 adverse events (one neonatal death, three stillbirth, one congenital, and six premature birth), which contain one newborn was both premature birth and congenital syphilis, one newborn was both premature birth and neonatal death. In the TPPA positive group, there were 98 normal newborns and nine adverse events (one neonatal death, one stillbirth, and eight premature birth), among which there was one newborn was both premature birth and neonatal death. The number of premature births was significant more than still birth, congenital and neonatal death $(P<0.01$, Figure $1 \mathrm{~b}$ ). There were six death events and four congenital, which occupied $0.99 \%$ and $0.66 \%$ of the whole infants, respectively. The percentage of adverse outcome rate was highest in RPR $\geq 1: 8$ group (11.24\%), followed with the TPPA positive group (8.41\%), and then the RPR $<1: 8$ group (2.56\%). However, the statistical difference was not significant $(P=0.09)$ in normal newborns and adverse events among RPR $<1: 8, R P R \geq 1: 8$ and TPPA positive groups (Table 3 ).

We further analyzed the correlation coefficient between adverse outcomes and pregnant women age, adverse events and RPR titers when giving birth. The result showed that adverse outcomes failed to collected with the pregnant age and PRP titers at giving birth (Table 4).

We calculated the pregnant women accepted benzathine penicillin $G$ for one course $(n=101)$, benzathine penicillin $G$ for one course and cephalosporin for one course $(n=18)$, benzathine penicillin $G$ for two courses $(n=210)$, benzathine penicillin $G$ for two course and cephalosporin for two courses $(n=26)$, benzathine penicillin $\mathrm{G}$ for three courses $(n=17)$ and 53 women without treatment. As shown in Table 3 , 
the treatments in RPR $\geq 1: 8$ group have a highest efficacy rate than RPR $<8$ group and TPPA positive group $(P<0.01)$. Patients accepted treatments have an efficacy rate of $65.60 \%$, which is higher than that with no treatment group (39.62\%, Table 5, Figure 2). The syphilis infected pregnant women accepted difference treatments were more efficacy $(P<0.01$, Table 5) compared with those without treatment.

\section{Discussion}

Although being easily detectable and treatable during pregnancy for many years, syphilis remains an important cause of perinatal death [13]. Pregnancy with syphilis could be transmitted to the fetus through cord blood and placenta, causing terrible harm to children. Worse, mothers with high RPR baseline titers, refused to receive treatments, and later treatments are most at risk of delivering an adverse outcome [23]. Pregnancy syphilis often has no obvious clinical symptoms, and latent syphilis accounts for the vast majority. This may be related to the temporary inhibition of the immune system in pregnant women during pregnancy. However, no matter it was active syphilis or latent syphilis, miscarriage, premature birth, stillbirth, congenital syphilis, and even neonatal death could happen [24]. The result of our study revealed that maternal syphilis could cause extremely high risk of adverse outcome events, especially premature birth, stillbirth and congenital syphilis.

The results in our study indicated that perinatal adverse outcome is closely related to the RPR titer baseline of their mothers. Additionally, high RPR baseline together with none treatment could increase the risk of adverse perinatal outcome [14]. We also found that the infection degree of mothers directly influenced it of their babies to a certain degree, with the trending of higher the titer of RPR in mother, higher RPR titer and adverse pregnancy outcome in their infants. The RPR seropositive rate at birth is significantly higher in RPR $\geq 1: 8$ group than $R P R<8$ group and TPPA positive group, which needed to be highly valued that when the RPR titer rises above $1: 8$, the possibility of adverse pregnancy outcome and high RPR titer of the newborn should be informed, especially when the treatment is not effective. Not only that, we also found that double negative (RPR $=0$ and TPPA negative) newborns at 12 months decreased extremely if they have higher RPR titers, especially those obtain RPR $\geq 8$. None of the newborns with RPR $\geq 8$ turned double negative. In the study in Tanzania, they revealed that Tanzanian women who were RPR negative and TPHA positive or who had low RPR titers and a positive specific treponemal test result, didn't increased the risk of adverse birth outcomes when compared with seronegative women; but they demonstrated that the adverse outcome mainly occurred in 1:32 RPR [13].

Among all the possible adverse outcomes, neonatal death, premature delivery, congenital syphilis and stillbirth occurred frequently. The perinatal deaths represented $0.99 \%$ in our study encounter with syphilis, which was higher than $0.4 \%$ of all pregnancies in Beijing in the 2013-2015 period [14]. The incidence of neonatal death and stillbirth increased in the pregnant women with RPR titer $\geq 1: 8$, compared with those in RPR $<1: 8$. We sought that the number of enrolled samples should response for the insignificant difference. Moreover, the frequency of congenital syphilis was $0.66 \%$ during the study period, compared with 3.48\% among treated mothers from Shenzhen Province [20], 0.90\% from Beijing [14] in recent years. 
Most of the factors assumed as potential confounders in our study failed to alter the relationship between adverse outcomes and maternal age, and high-titer active syphilis at birth. The result in our study was consistent with the study in Tanzania [13].

For the terrible consequence of high RPR titer in syphilis pregnancy women, adequate treatment should be implemented once discovered. Timely diagnosis and early definitive penicillin treatment are the most important components of syphilis management during pregnancy $[25,26]$. Many researches have revealed that untreated syphilis in pregnancy carries significant risk for stillbirth or fetal loss, premature birth, low birth weight, congenital syphilis and neonatal death $[10,27,28]$. Studies consistently show that the earlier the treatment starts, the lower the risk of adverse pregnancy outcomes [26]. The risk of congenital syphilis could be raised by approximately $10 \%$ if there was one-week delay [29]. However, there were only $65 \%$ of the women had been treated adequately in the study of Zhang et al [14]. Delightful, the number of patients accepted treatment increased, with proportion of $84.17 \%$ in our study. However, the patients without treatment still occupied larger number, which highlighting the need to improve the implementation of the Chinese guidelines.

In the study of Moline et al [30], penicillin was considered as the only known effective treatment during pregnancy. However, some studies have put focus on alternative treatment on syphilis, including ceftriaxone, amoxicillin, and doxycycline [31-34]. In our study, penicillin is the first choice for anti-syphilis, except those allergic patients. In the study of Liang et al [21] they revealed that ceftriaxone compared with penicillin for the treatment of syphilis has insignificant effective. The result in our study revealed that the effect increased with the addition of courses, and the effect achieve best when combined with ceftriaxone. In addition, we also found that the higher the RPR titer was, the more efficacy it could be. Regrettably, the intention-to-treat patients enrolled in our study is still not enough.

\section{Conclusions}

In summary, our study revealed that syphilis continues to affect many pregnancy infected women and their infants in recent years, especially those with high RPR titers. Persistent infections with high baseline syphilis RPR are an important cause of congenital syphilis, neonatal death, stillbirth, and premature birth, especially in patients with RPR titers of $\geq 1: 8$. Therefore, RPR titer at 1:8 could be considered as a point of therapeutic induction. Although the rate of patients accepted treatment increased, but there are still many women who had not been detected or treated in time. Among the patients accepted treatments, 2course performed better than 1-course. The more the RPR titer was, the more efficacy it was. In addition, early detection and treatment can reduce the risk reduction, so it is emphasized that early detection and standardized treatment are the only effective means to prevent mother-to-child transmission of syphilis.

\section{Declarations}

Acknowledgements 
Upon the completion of the thesis, I would like to take this opportunity to express my sincere gratitude to my supervisor, who has given me important guidance on the thesis. I am also grateful to all the members of my department of Gynecology and Obstetrics, who have provided me with a lot of help and guidance.

\section{Funding}

This study was supported without funding.

\section{Availability of data and materials}

Datasets used and analyzed during the current study are available from the corresponding author on reasonable request. All personal identifiers were removed from the dataset.

\section{Author information}

\section{Affiliations}

${ }^{1}$ Department of Gynecology and Obstetrics, The second Affiliated hospital of the Southeast University, Nanjing, 210003, Jiangsu, China

\section{Corresponding author:}

Correspondence to Genju Wang

\section{Authors' contribution}

Project development: GW, GH; data collection: YJ, YD; Data analysis, GW, HJ, JT; Manuscript writing: GW;

\section{Ethics declarations}

\section{Ethical approval and consent to participate}

This study was permitted by the Human Ethics Committee of the Nanjing Second Hospital.

\section{Consent for publication}

The local ethics committee waived the necessity for individual informed consent.

\section{Competing interests}

The authors declare that they have no competing interests.

\section{References}

1. The Chinese medical association branch of obstetrics and gynecology infectious diseases group: Expert Consensus on Pregnancy with Syphilis Diagnosis and Treatment. Chinese J Obstet Gynecol 
47, 158-160 (2012)

2. Ameeta, E.S, Karen, S., Bonita, L., Joan, L.R., Tom, W.: Resurgence of early congenital syphilis in Alberta. Can Med Assoc J 177 (1), 33-36 (2007)

3. Sriparna, B., Ashok, K.: Varied presentations of early congenital syphilis. J Trop Pediatr 59, 250-254 (2013)

4. Wang, A.L., Qiao, Y.P., Wang, L.H., Fang, L.W., Wang, F., Jin, X., Qiu, J., Wang, X.Y., Wang, Q., Wu, J.L., Sten, H.V., Song, L.: Integrated prevention of mother-to-child transmission for human immunodeficiency virus, syphilis and hepatitis B virus in China. Bull World Health Organ 93, 52-56 (2015)

5. Dou, L., Wang, X., Fang, W., Qian, W., Qiao, Y., Min, S., Xi, J., Jie, Q., Li, S., Wang, A.: Epidemic Profile of Maternal Syphilis in China in 2013. BioMed Res Int 2016, 1-8 (2016)

6. Dang, Q., Feng, J., Lu, X., Zhang, X., Xu, H., Liu, C., Nu, X.: Evaluation of specific antibodies for early diagnosis and management of syphilis. Int J Dermatol 45, 1169-1171(2010)

7. Yang, L.G., Tucker, J.D., Wang, C., Shen, S.Y., Chen, X.S., Yang, B., Peeling, R.: Syphilis Test Availability and Uptake at Medical Facilities in Southern China. Bull World Health Organ 89, 798-805 (2011)

8. Chuck, A., Ohinmaa, A.P., Singh, A., Jacobs, P.: Cost effectiveness of enzyme immunoassay and immunoblot testing for the diagnosis of syphilis. Int J Std Aids 19, 393-399 (2008)

9. Kojima, N., Bristow, C., Maecker, H., Rosenberghasson, Y., Leon, S., Rivera, S.V., Konda, K., Klausner, J.: A Pilot Study of 63 Cytokines in Patients With and Without Syphilis in Lima, Peru. Open Forum Infect Di 2, 883 (2015)

10. Gomez, G.B., Kamb, M.L., Newman, L.M., Jennifer, M., Nathalie, B., Hawkes, S.J.: Untreated maternal syphilis and adverse outcomes of pregnancy: a systematic review and meta-analysis. Bull World Health Organ 91, 217-226 (2013)

11. Rac, M.W.F., Revell, P.A., Eppes, C.S.: Syphilis during pregnancy: a preventable threat to maternal-fetal health. Am J Obstet Gynecol 216, 352-363 (2018)

12. Lin, J.S., Eder, M.L., Bean, S.I.: Screening for Syphilis Infection in Pregnant Women: Updated Evidence Report and Systematic Review for the US Preventive Services Task Force. Obstet Gynecol Survey (2019)

13. Deborah, W.J., John, C., Balthazar, G., Helen, W., Mary, R., Leonard, N., Anne, W., Rebecca, B., James, T., Donatila, N.: Syphilis in pregnancy in Tanzania. I. Impact of maternal syphilis on outcome of pregnancy. J Infect Dis 186, 940-947 (2002)

14. Zhang, X., Yu, Y., Yang, H., Xu, H., Sten, H.V., Liu, K.: Surveillance of Maternal Syphilis in China: Pregnancy Outcomes and Determinants of Congenital Syphilis. Med Sci Monit 24, 7727-7735 (2018)

15. Ebenezer, E.D., Benjamin, S.J., Sahni, R.D., Prakash, J.A., Chelliah, H., Mathews, J.E.: A retrospective study of the prevalence and outcomes of syphilis in pregnancy in a five-year period. Int $\mathrm{J}$ Gynaecol Obstet 140, 42-46 (2018) 
16. Yang, C.J., Lee, N.Y., Chen, T.C., Lin, Y.H., Liang, S.H., Lu, P.L., Huang, W.C., Tang, H.J., Lee, C.H., Lin, H.H., Chen, Y.H., Ko, W.C., Hung. C.C.: One Dose versus Three Weekly Doses of Benzathine Penicillin G for Patients Co-Infected with HIV and Early Syphilis: A Multicenter, Prospective Observational Study. Plos One 9, e109667 (2014)

17. Douglas, J.M.: Penicillin Treatment of Syphilis Clearing Away the Shadow on the Land. Jama- J Am Med Assoc 301, 769-771 (2009)

18. Workowski, K.A.: Centers for Disease Control and Prevention sexually transmitted diseases treatment guidelines. Clin Infect Dis 61(suppl_8), 759-762 (2015)

19. Kingston, M., French, P., Goh, B., Goold, P., Higgins, S., Sukthankar, A., Stott, C., Turner, A., Tyler, C., Young, H.: UK National Guidelines on the Management of Syphilis 2008. Int J Std Aids 19, 729-740 (2008)

20. Hong, F.C., Wu, X.B., Yang, F., Lan, L.N., Guan, Y., Zhang, C.L., Feng, T.J., Yang, Y.Z., Yin, Y.P., Yu, W.Y.: Risk of Congenital Syphilis (CS) Following Treatment of Maternal Syphilis: Results of a CS Control Program in China. Clin Infect Dis 65, 588-594 (2017)

21. Liang, Z., Chen, Y.P., Yang, C.S., Guo, W., Jiang, X.X., Xu, X.F., Feng, S.X., Liu, Y.Q., Jiang, G.: Metaanalysis of ceftriaxone compared with penicillin for the treatment of syphilis. Int J Antimicrob Ag 47, 6-11 (2016)

22. Shann, S., Wilson, J.: Treatment of neurosyphilis with ceftriaxone. Sex Transm Infect 79, 415-416 (2003)

23. Qin, J.B., Feng, T.J., Yang, T.B., Hong, F.C., Lan, L.N., Zhang, C.L., Yang, F., Mamady, K., Dong, W.: Risk Factors for Congenital Syphilis and Adverse Pregnancy Outcomes in Offspring of Women With Syphilis in Shenzhen, China. Sex Transm Dis 41, 13-23 (2014)

24. Krakauer, Y., Pariente, G., Sergienko, R., Wiznitzer, A., Sheiner, E.: Perinatal outcome in cases of latent syphilis during pregnancy. Int J Gynaecol Obstet 118, 15-17 (2012)

25. Tucker, J.D., Cohen, M.S.: China's syphilis epidemic: epidemiology, proximate determinants of spread, and control responses. Curr Opin in Infect Dis 24, 50-55 (2011)

26. Qin, J.B., Feng, T.J., Yang, T.B., Hong, F.C., Lan, L.N., Zhang, C.L., Liu, X.L., Yang, Y.Z., Xiao, S.Y., Tan, H.Z.: Synthesized prevention and control of one decade for mother-to-child transmission of syphilis and determinants associated with congenital syphilis and adverse pregnancy outcomes in Shenzhen, South China. Eur J Clin Microbiol Infect Dis 33, 2183-2198 (2014)

27. Qin, J., Yang, T., Xiao, S., Tan, H., Feng, T., Fu, H.: Reported estimates of adverse pregnancy outcomes among women with and without syphilis: a systematic review and meta-analysis. Plos One $\mathbf{9}$, e102203 (2014)

28. Braccio, S., Sharland, M., Ladhani, S.N.: Prevention and treatment of mother-to-child transmission of syphilis. Curr Opin Infect Dis 29, 268-274 (2016)

29. Liu, J.B., Hong, F.P., Zhou, H., Yang, F., Cai, Y.M., Wen, L.Z., Lai, Y.H., Lin, L.J., Zeegers, M.P.: A risk model for congenital syphilis in infants born to mothers with syphilis treated in gestation: a prospective cohort study. Sex Transm Infect 86, 292-296 (2010) 
30. Moline, H.R., Jr, S.J.: The continuing threat of syphilis in pregnancy. Curr Opin Obstetr Gynecol 28, 101-104 (2016)

31. Cao, Y., Su, X., Wang, Q., Xue, H., Zhu, X., Zhang, C., Jiang, J., Qi, S., Gong, X., Zhu, X.A.: Multicenter Study Evaluating Ceftriaxone and Benzathine Penicillin $G$ as Treatment Agents for Early Syphilis in Jiangsu, China. Clin Infect Dis 65, 1683-1688 (2017)

32. Katanami, Y., Hashimoto, T., Takaya, S., Yamamoto, K., Kutsuna, S., Takeshita, N., Hayakawa, K., Kanagawa, S., Ohmagari, N.: Amoxicillin and Ceftriaxone as Treatment Alternatives to Penicillin for Maternal Syphilis. Emerg Infect Dis 23, 827-829 (2017)

33. Drago, F., Ciccarese, G., Broccolo, F., Sartoris, G., Stura, P., Esposito, S., Rebora, A., Parodi, A.: A new enhanced antibiotic treatment for early and late syphilis. J Glob Antimicrob Re 5, 64-66 (2016)

34. Hook, E.W., Frieda, B., Kathleen, V.D., Noro, R., Peter, L., Sena, A.C., David, M., Carol, L., Linda, M.N., Mark, W.: A phase III equivalence trial of azithromycin versus benzathine penicillin for treatment of early syphilis. J Infect Dis 201, 1729-1735 (2010)

\section{Tables}

Table 1. The relationship among pregnant women age and the RPR titer of pregnant, total RPR titer of newborns and total adverse outcomes.

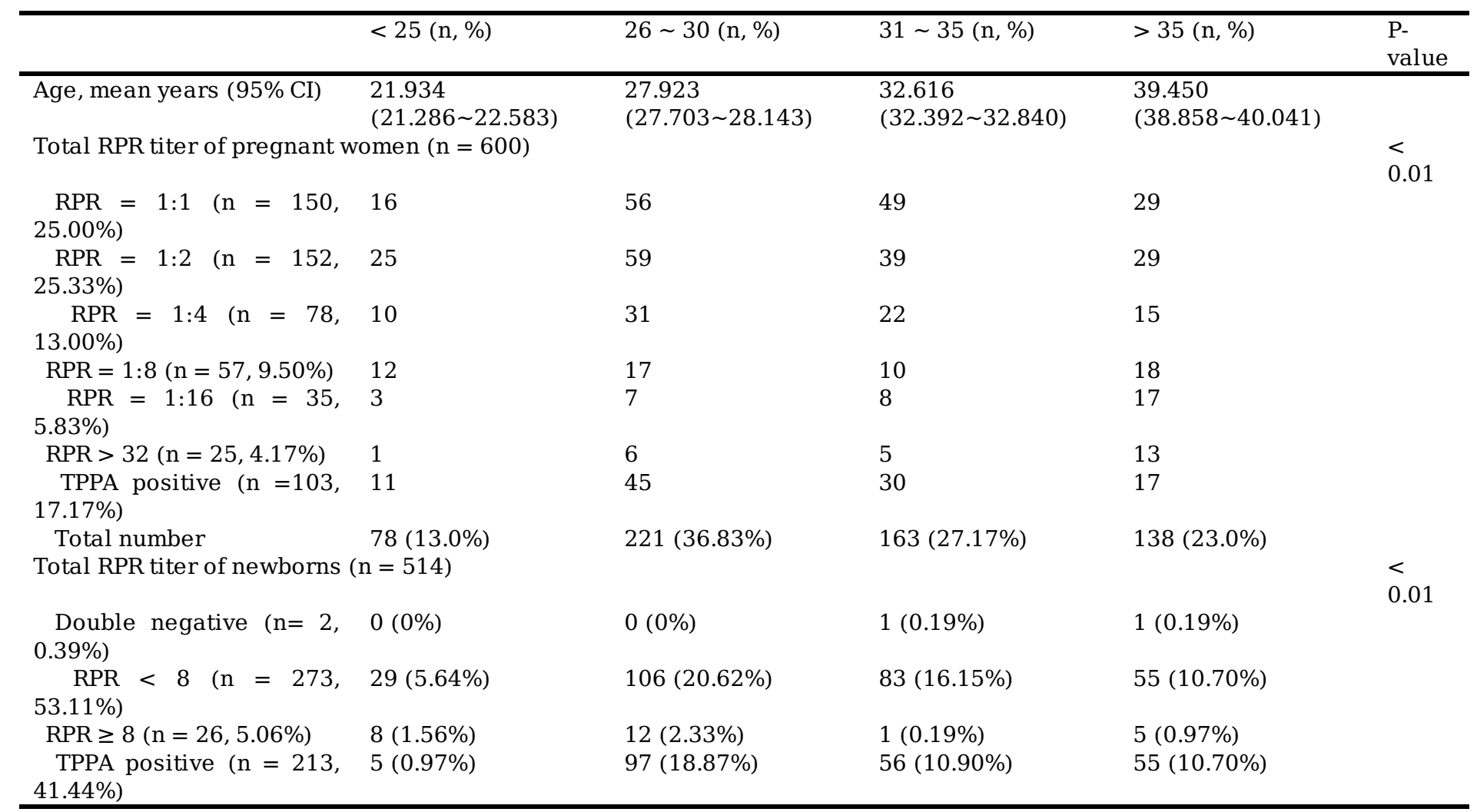

Table 2.Relationship between RPR titer of syphilis before delivery, the RPR titers of the newborns and negative conversion rate after 12 months of born $(n=587)$ 


\begin{tabular}{|c|c|c|c|c|c|c|c|c|c|c|c|}
\hline \multirow{2}{*}{$\begin{array}{l}\text { RPR titers of } \\
\text { pregnant women }\end{array}$} & \multirow{2}{*}{$\begin{array}{l}\text { Number of } \\
\text { pregnant women }\end{array}$} & \multicolumn{9}{|c|}{ RPR titers of newborns } & \multirow[b]{2}{*}{$\begin{array}{l}\text { Double } \\
\text { negative } \\
\text { after } 12 \\
\text { months of } \\
\text { born }\end{array}$} \\
\hline & & $\begin{array}{l}\text { Double } \\
\text { negative }\end{array}$ & $\begin{array}{l}\text { TPPA } \\
\text { positive }\end{array}$ & $1: 1$ & $1: 2$ & $1: 4$ & $1: 8$ & $1: 16$ & $>1: 32$ & Unknown & \\
\hline TPPA positive & 106 & 2 & 92 & 3 & 2 & 0 & 0 & 0 & 0 & 7 & 52 \\
\hline $1: 1$ & 152 & 1 & 84 & 58 & 5 & 0 & 0 & 0 & 0 & 4 & 67 \\
\hline $1: 2$ & 156 & 0 & 48 & 60 & 41 & 4 & 0 & 0 & 0 & 3 & \\
\hline $1: 4$ & 84 & 0 & 8 & 28 & 36 & 10 & 0 & 0 & 2 & 0 & \\
\hline $1: 8$ & 57 & 0 & 2 & 6 & 20 & 17 & 6 & 1 & 1 & 4 & 0 \\
\hline $1: 16$ & 23 & 0 & 0 & 2 & 5 & 8 & 4 & 2 & 0 & 2 & \\
\hline$\geq 1: 32$ & 9 & 0 & 0 & 0 & 2 & 1 & 2 & 2 & 1 & 1 & \\
\hline
\end{tabular}

Table 3. The relationship between RPR titer of syphilis infect pregnant women and their newborns weight and adverse outcomes.

\begin{tabular}{|c|c|c|c|c|}
\hline Items & $<1: 8$ & $\geq 1: 8$ & TPPA positive & P-value \\
\hline$\overline{\text { Age (years) }}$ & $31.24(30.63 \sim 31.84)$ & $30.71(29.58 \sim 31.84)$ & $32.22(31.03 \sim 33.41)$ & $<0.01$ \\
\hline Newborn weight (g) & $3363.7(3315.4 \sim 3411.9)$ & $3201.6(3074.4 \sim 3328.7)$ & $3241.3(3111.4 \sim 3371.2)$ & $>0.05$ \\
\hline Adverse pregnancy outcomes & & & & $>0.09$ \\
\hline Total adverse events (n, \%) & $10(2.55)$ & $10(11.24)$ & $9(8.41)$ & \\
\hline Neonatal death $(\mathrm{n})$ & 0 & 1 & 1 & \\
\hline Stillbirth (n) & 0 & 3 & 1 & \\
\hline Therapy & & & & $<0.01$ \\
\hline Efficacy (n, \%) & $96(38.71)$ & 169 (89.89) & $1(12.5)$ & \\
\hline no response $(\mathrm{n}, \%)$ & $152(61.29)$ & $19(10.11)$ & $7(87.5)$ & \\
\hline
\end{tabular}

a 9 premature birth contain 1 congenital syphilis

b 6 premature birth contain 1 congenital syphilis and 1 neonatal death

C 8 premature contain 1 neonatal death

Table 4. Logistic analysis among adverse pregnancy outcomes and pregnant age, and RPR titers when giving birth

\begin{tabular}{llllll}
\hline & B & P-value & $\operatorname{Exp}(\mathrm{B})$ & \multicolumn{2}{c}{ 95\% EXP (B) } \\
\cline { 5 - 6 } & & & & Lower & Upper \\
\hline Age & -0.039 & 0.298 & 0.962 & 0.894 & 1.035 \\
RPR titers* & 0.113 & 0.702 & 1.120 & 0.628 & 1.997 \\
\hline
\end{tabular}

* The RPR titers when giving birth.

Table 5. Effects of different therapy methods in pregnant women $(n=425)$ 


\begin{tabular}{|c|c|c|c|c|}
\hline Therapy method & Numbers & $\begin{array}{l}\text { Efficacy a (n, } \\
\%)\end{array}$ & $\begin{array}{l}\text { No response }{ }^{b}(\mathrm{n}, \\
\%)\end{array}$ & $\begin{array}{l}\mathrm{P}- \\
\text { value }\end{array}$ \\
\hline No therapy & 53 & $21(39.62)$ & $32(60.38)$ & $<0.01$ \\
\hline Total number of accepted therapies & 372 & $244(65.60)$ & $128(34.40)$ & \\
\hline Benzathine penicillin $\mathrm{G}$ for one course & 101 & $72(71.29)$ & $29(28.71)$ & $<0.01$ \\
\hline $\begin{array}{l}\text { Benzathine penicillin } \mathrm{G} \text { for one course and cephalosporin for one } \\
\text { course }\end{array}$ & 18 & $14(77.78)$ & $4(22.22)$ & \\
\hline Benzathine penicillin G for two courses & 210 & $124(59.05)$ & $86(40.95)$ & \\
\hline $\begin{array}{l}\text { Benzathine penicillin } \mathrm{G} \text { for two courses and cephalosporin for one } \\
\text { course }\end{array}$ & 26 & $21(80.77)$ & $5(19.23)$ & \\
\hline Benzathine penicillin $\mathrm{G}$ for three courses & 17 & $13(76.47)$ & $4(23.53)$ & \\
\hline
\end{tabular}

$\mathrm{a}$ the RPR titer was decreased after therapy.

$\mathrm{b}$ the RPR titer was either increased or not changed after therapy.

\section{Figures}


RPR $<1: 8$

$R P R \geq 1: 8$

RPR negative and TPPA positive

a

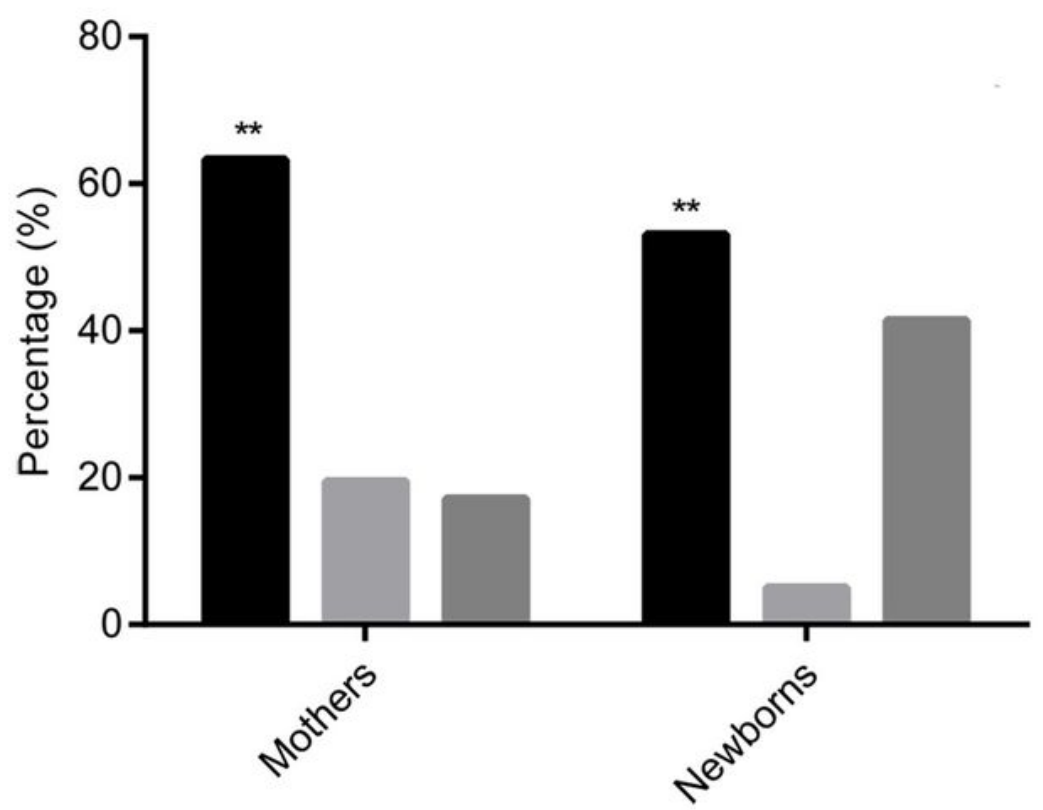

b

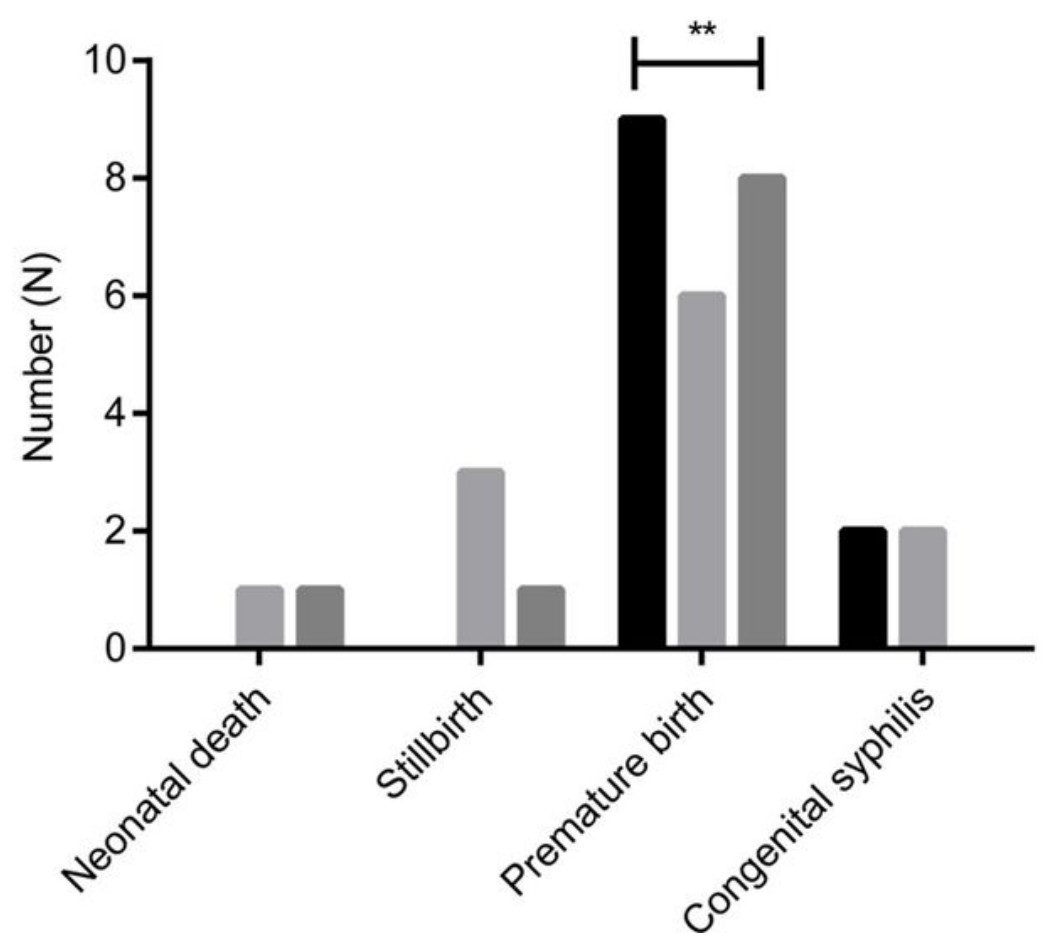

Figure 1

The RPR titer and adverse outcomes of newborns distribution. a囚The comparison of RPR titer distribution in mothers and newborns. ** represented the percentage of mothers and newborns in RPR $<1: 8$ group was significantly higher than RPR $\geq 1: 8$ and RPR negative and TPPA positive groups. b $\otimes T$ he adverse events in different RPR titer groups $(P<0.01)$. ** represented the premature birth were significantly higher than neonatal death, stillbirth, and congenital syphilis groups in RPR $<1: 8, R P R \geq 1: 8$ and RPR negative 
and TPPA positive groups $(P<0.01)$. RPR, Rapid Plasma Reagin; TPPA, Treponema pallidum particle agglutination.

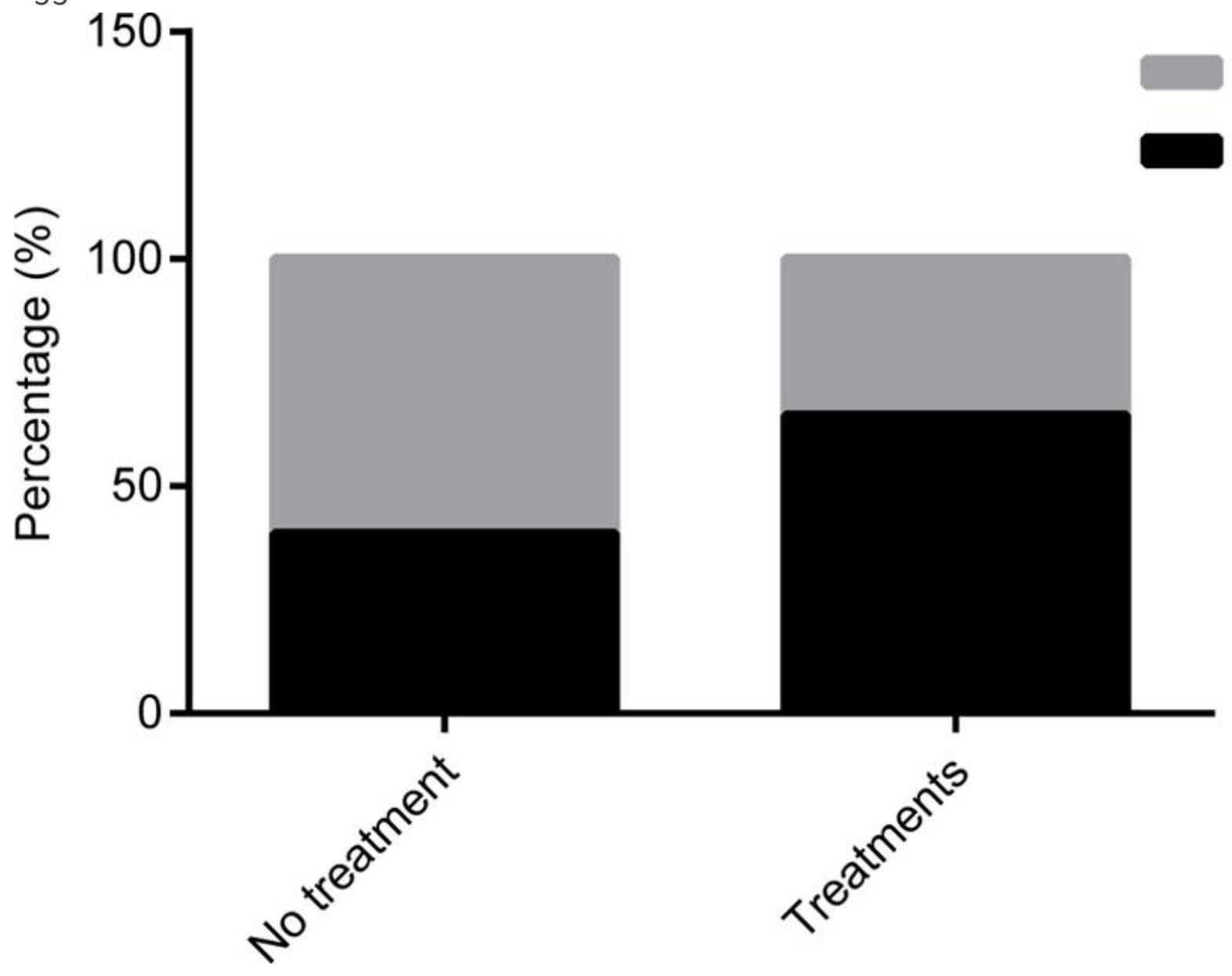

Figure 2

The comparison of effects in groups with treatments and without treatment. 